\title{
An Optimized Watermarking Technique Using Wavelet Packet and Singular Value Decomposition
}

\author{
Bashar Ahmed Sahar Q. Saleh \\ Department of Mathematics and computer science \\ Faculty of science, Alexandria University \\ Alexandria, Egypt \\ Bashar.hozaim@gmail.com \\ sahar.qasim2009@gmail.com
}

\author{
Khaled M. Mahar \\ Department of Computer Science \\ Collage of Computing and Information Technology \\ Arab Academy for Science \& Technology and Maritime \\ transport, Alexandria, Egypt \\ khmahar@aast.edu
}

\begin{abstract}
This paper exploits the characteristics of both wavelet packet transform and singular values decomposition to propose a high capacity and robust watermarking technique. From the wavelet packets, the best wavelet sub-band, in the sense of evaluation metric, is found by using the particle swarm optimization algorithm to meet the requirements of imperceptibility and robustness of the watermarking algorithm. After the operation of particle swarm optimization, the watermark image can be embedded into the cover image, and the watermarked image is obtained. Intentional signal processing or malicious attacks are expected, and the watermarking algorithm is considered to keep the integrity for the media file. Experimental results demonstrate that the proposed method can increase the capability to resist such attacks. Moreover, the watermarked image quality can be guaranteed based on the inherent characteristics of the singular values decomposition concept.
\end{abstract}

Keywords-Wavelet packets transform; singular values decomposition; particle swarm optimization; images watermarking; imperceptibility; robustness;

\section{INTRODUCTION}

Due to the rapid growth of Internet and communication media, the availability of digital multimedia content has increased in fast and has become more suitable for exchange the digital information. Consequently, many applications have also raised concern about copyright issues, illegal change and delivery of digital information. Watermarking technology is used to resolve the issue of integrity and trusted communication. Watermarking [1] is the technique of embedding data into a multimedia content such as video, audio, image file. The embedded data can be extracted from the multimedia file to recognize the owner of the copyright. Cryptography, steganography are other techniques besides the watermarking that can also keep the digital content [2].

Watermarking and steganography both are data hiding techniques which conceal secret data in the cover work. But there is a small difference between them. Steganography conceals the presence of secret data. When the presence of secure data is discovered, the steganography fails. However, the existence of secret data in watermarking can be identified [3]. Ideally, the aim of watermarking is to make the manipulation /removal of secure data hard. Cryptography in contrast does not hide the existence of secure data but it encrypts the data in such a technique which it seems useless to a pirate if decrypted with the suitable key $[4,5]$.

A basic watermarking algorithm for example consists of a host image, a watermark structure, an inserting method, and an extraction method. Many techniques have been proposed in the literature for protecting the digital images $[1,2,6,7]$.

The watermark embedding techniques are separated into two groups: spatial-domain and frequency-domain methods. Inserting the watermark into the spatial-domain of the cover image has the advantages of easy implementation and low computational time. But these methods are commonly fragile to different attacks. On the other hand, the frequency-domain techniques insert the watermark in the transform coefficients such as discrete wavelet transform (DWT), wavelet packet transform (WPT), discrete Fourier transform (DFT), finite ridgelet transform (FRT) and discrete cosine transform (DCT) [8-12]. Frequency-domain approaches also embed more information and they are more robust against different types of attacks but they have more computational time than spatialdomain watermarking approaches.

According to the robustness, the watermark extraction can be categorized into three groups: namely robust, semi fragile and fragile [13]. Robust watermarking methods is designed to keep the watermark image if the watermarked image undergoes different attacks while the watermark is fragile if the watermarking methods is not capable to resist intentional or signal processing attacks. So, robust watermarking methods are suitable for digital media authentication [14, 15]. However, watermark in semi-fragile watermarking methods requires surviving slight alterations. Any one of these three types of watermarking methods can be used according to the nature of the application under study.

For watermarking scheme, different applications have many requirements to be satisfied but it is quite difficult to have a unique set of requirements. The requirements regarding the rightful ownership and copyright protection are as follows [16]. Robustness denotes to the watermark ability to be kept even after some modifications presented by intentional and unintentional data processing. These modifications are also 
called watermarking attacks. Imperceptibility denotes to the watermark perceptual transparency. In other words, watermark must be inserted in such a way that the human vision system unable to notice differences between the original and the watermarked media. Capacity denotes to maximizing the amount of data that can be concealed in the media. This directly affects the perceptual transparency and robustness. Security denotes to the watermark cannot be discovered or read by the unauthorized person; but, the authorized user should correctly recover the watermark. These requirements contradict with each other so a trade-off among them is needed. There are different watermarking schemes in transform domain and in spatial domain that have addressed the issues of the watermarking requirements and make efforts to balance between these inconsistent requirements [17-19].

The remainder of this paper is organized as follows. Wavelet Packet Transform, Singular Value Decomposition (SVD) and Particle Swarm Optimization (PSO) are briefly introduced in section 2. In section 3, the proposed watermarking techniques are presented. The experimental results and are shown and discussed in section 4. Finally, section 5 concludes this paper with some remarks to future work.

\section{BACKGROUND REVIEW}

\section{A. Wavelet Packet Transform}

WPT is a generalization to the discrete wavelet transform. Wavelet transform decomposes the signal in narrow frequency bands while preserving the basis signals space limited [20]. Only low-pass filtered sub-band is recursively decomposed. When the level is decomposed further, the complexity will be increased and the average image will be more stable. In wavelet transform, if an original image is decomposed into two levels, the result will be an average image LL1 and three detail images LH1, HL1 and HH1 in level one. Then wavelet analysis is used on the average image LL1 again to get four lower resolution sub-band images LL2 and three detail images LH2, HL2 and HH2. The average image LL2 holds the most information of the original image but others hold some highfrequency [21]. The watermark can be inserted into $\mathrm{LH} 2$ or HL2 but it is not inserted into LL2 due to the serious degradation of image quality which is the main drawback of wavelet transform. Fig.1. shows wavelet transform tree structure of image decomposition into two levels. In wavelet packets, all sub-bands LL1, LH1, HL1 and HH1 (average image and the three detail images) are decomposed as demonstrated in Fig.2. The benefits of these further sequences of decomposition are the time frequency plane is split more accurately and the amount of inserting information will be increased. 16 sub-bands of coefficients are generated using two level wavelet packet transform. Further decomposition to subband offers more time resolution and thus raises the imperceptibility and robustness of the watermarking method.

\section{B. Singular Values Decomposition}

SVD is an effective tool for analyzing matrices. A digital image can be considered as a square or rectangular matrix of non-negative scalar entries. The SVD of a digital image $I$ with

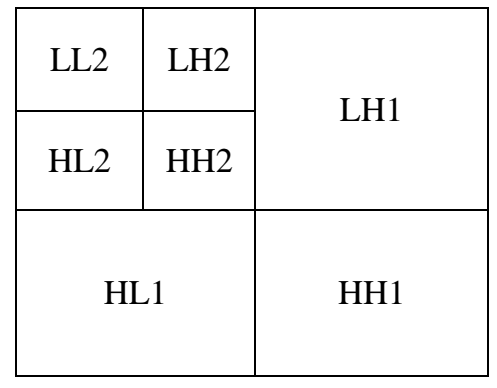

Fig. 1. The structure of two level wavelet transform.

\begin{tabular}{|l|l|l|l|}
\hline LLA2 & LLH2 & LHA2 & LHH2 \\
\hline LLV2 & LLD2 & LHV2 & LHD2 \\
\hline HLA2 & HLH2 & HHA2 & HHH2 \\
\hline HLV2 & HLD2 & HHV2 & HHD2 \\
\hline
\end{tabular}

Fig. 2. The structure of two level wavelet packet transform.

size $M \times N$ where $\mathrm{M} \leq N$ is the factorization of $I$ into three matrices, $U, S$ and $V$.

$$
\mathrm{I}=\mathrm{U}^{*} \mathrm{~S}^{*} \mathrm{~V}^{\mathrm{T}}
$$

where $U$ and $V$ are orthogonal matrices and $S$ is a diagonal matrix of singular values $\mho_{j} ;=1,2, \ldots, M$ whose diagonal entries satisfy:

$$
\gamma_{1} \geq \gamma_{2} \geq \gamma_{3} \geq \ldots \geq \gamma_{r}>\gamma_{r+1=\ldots}=\gamma_{m}=0
$$

where $r$ is the rank of $I$, that equals the number of non-zero singular values in $S$. The diagonal inputs of $S$ are positive square roots of the eigenvalues of $I I^{T}$ or $I^{T} I$, and are namely the singular values of $I$. The left singular vectors of $I$ are the $U$ columns (eigenvectors of $I I^{T}$ ). The right singular vectors of $I$ are the $V$ columns (eigenvectors of $I^{T} I$ ). The watermark image can be inserted into cover image by modifying its singular values due to the main properties of SVD. Singular values represent intrinsic algebraic properties. When a small modification is added to an image, large variation of its singular values does not occur and will not affect the image visual properties [22].

\section{Particle Swarm Optimization}

Ideally, the PSO algorithm exchanges the information of the local and global best values [23]. Assume that the optimization goal is to maximize an objective function $f(r)$. Each potential solution is given a velocity randomly, and the potential solutions correspond to individuals. Each particle (individual) in PSO flies in the d-dimensional problem space with a velocity which is dynamically adjusted corresponding to the flying experiences of its individuals and their classmates. 
The location of the $i^{\text {th }}$ particle is represented as $X_{i}=\left[x_{1}\right.$, $\left.x_{2}, \ldots, x_{d}\right]$. The best previous position (which gives the best fitness value) of the $i^{\text {th }}$ particle is recorded and represented as $P_{i}=\left[p_{1}, p_{2}, \ldots, p_{d}\right]$, which is also called $p_{\text {best }}$. The index of the best particle among all the particles in the population is represented by this symbol. The location $p_{g}$ is also denoted by $g_{\text {best }}$. The velocity of the $i^{t h}$ particle is represented by $V_{i}=\left[v_{1}, v_{2}, \ldots, v_{d}\right]$, where $v_{i}$ takes the values from the interval [0, 255]. The particle swarm optimization concept resides on, at each time step, regulating the velocity and location of each particle toward its $p_{\text {best }}$ and $g_{\text {best }}$ locations according to:

$$
\begin{gathered}
\mathrm{V}_{\mathrm{i}}^{\mathrm{t}+1}=\mathrm{wV}_{\mathrm{i}}^{\mathrm{t}}+\mathrm{c}_{1} \mathrm{r}_{1}\left(\mathrm{p}_{\mathrm{i}}+\mathrm{X}_{\mathrm{i}}^{\mathrm{t}}\right)+\mathrm{c}_{2} \mathrm{r}_{2}\left(\mathrm{p}_{\mathrm{g}}+\mathrm{X}_{\mathrm{i}}^{\mathrm{t}}\right) \\
\mathrm{X}_{\mathrm{i}}^{\mathrm{t}+1}=\mathrm{X}_{\mathrm{i}}^{\mathrm{t}}+\mathrm{V}_{\mathrm{i}}^{\mathrm{t}+1}
\end{gathered}
$$

where ' $i$ ' denotes the number of the particle in the swarm. ' $r$ ' and ' $r$ ' are the random values in velocity updating processes. ' $p_{i}$ ' and ' $p_{g}$ ' are denoted the location of the best problem solution vector found by ' $i$ ' and the location of the best particle (solution) among all the particles in the population, respectively. ' $w$ ', ' $c_{1}$ ' and ' $c_{2}$ ' (user-supplied coefficients parameters) have default values and defined as ' 1.2 ' for ' $w$ ' and ' 0.5 ' for ' $c_{1}$ ' and ' $c_{2}$ '. ' $w$ ' ensures convergence of the PSO algorithm.

Regarding the fitness function, two common performance evaluation metrics of the images are combined to form the fitness function; the first is the Peak Signal to Noise Ratio $\left(\mathrm{PSNR}_{\mathrm{im}}\right)$ between the original cover image and the watermarked image to measure the imperceptibility. The second is the Peak Signal to Noise Ratio $\left(\mathrm{PSNR}_{\mathrm{ro}}\right)$ between the original watermark image and the extracted watermark image to measure the robustness as suggested by [22]. The fitness function is formed by combining the two metrics as shown in (5).

$$
\text { Fitness function }=\mathrm{PSNR}_{\text {im }}+\mathrm{PSNR}_{\mathrm{ro}}
$$

where $\mathrm{PSNR}_{\mathrm{im}}$ and $\mathrm{PSNR}_{\mathrm{ro}}$ are calculated using:

$$
\text { PSNR }=10 \times \log _{10}\left(\frac{255^{2}}{\frac{1}{N \times N} \sum_{i=1}^{N} \Sigma_{j=1}^{N}\left(I(i, j, j)-I^{I}(i, j, j)\right)^{2}}\right)
$$

\section{Proposed WATERMARKING TeChNiQUeS}

Any watermarking technique consists of two components, watermarking embedding and watermarking extraction. This work mainly use the wavelet packet transform. Some additional techniques such as SVD are also used to enhance the results of the watermarking algorithm. Those methods are described in the following sub sections.

\section{A. High Capacity Watermarking}

This section describes the simplest form of the proposed watermarking algorithm. It maximizes the amount of watermark data which can be concealed in the cover images in which the embedded watermark image size is equal to cover image size.

1) Watermark embedding: Suppose that original image $I$ is a gray-level image with size $M \times N$. The watermark $W$ is a binary image with size $X \times Y$. First, the original image is decomposed into the wavelet packet representation of m-level and obtain multi-resolution representation of the image. The watermark embedding process goes through the following steps:

- Use four-level Haar wavelet packet to decompose the cover image $I$ into 256 sub-bands in level 4.

- $\quad$ Divide the watermark image into 256 non-overlapped blocks: $W=W^{l}+W^{2}+\ldots+W^{256}$ where $W^{x}$ points to the watermark block.

- Modify the coefficients of each sub-band in the cover image with the values of each watermark block $C^{\prime x}=$ $C^{x}+\alpha W^{x}$ where $C^{x}$ denotes to the coefficients values of the cover sub-band $x, W^{x}$ denotes the values of the watermark block $x$ and $\alpha$ denotes to the scalar factor.

- Get the 256 sets of modified wavelet packet coefficients.

- Reconstruct the resultant sets of modified wavelet packet coefficients to get the watermarked image $I_{w}$.

2) Watermarking extraction: The watermark extraction is the reverse procedure of the watermark embedding. It can be summarized as follows:

- Use four-level Haar wavelet packet to decompose the watermarked image $I_{w}$ and the original image $I$ into 256 sub-bands in level 4.

- Extract the watermark blocks from each sub-band $W^{x}=\left(C^{x}-C^{x}\right) / \alpha$ where $x$ represents the sub-band or block $x=1,2, \ldots, 256, C^{\prime x}$ denotes the coefficients of the watermarked sub-band, $C^{x}$ points to the coefficients of the cover image sub-band and $\alpha$ denotes to the scalar factor.

\section{B. High capacity watermarking using WPT-SVD}

This method is a sophisticated version of the method presented in the previous section. The singular values decomposition technique is applied along with the wavelet packet transform. The watermark embedding and extraction processes are presented in the following sub sections.

1) Watermarking embedding: The watermark embedding process goes through the steps listed here:

- Use four-level Haar wavelet packet to decompose the cover image $I$ into 256 sub-bands in level 4.

- Apply SVD to each sub-band $F^{\tilde{T}}=U_{b}^{\pi} S_{b}^{\pi} V_{b}^{R T}$, $x=1,2, \ldots 256$ where $x$ represents the cover image subband.

- $\quad$ Divide the watermark image into 256 non-overlapped blocks: $W=W^{l}+W^{2}+\ldots+W^{256}$ where $W^{x}$ points to the watermark block.

- Apply SVD to each watermark block $W^{x}=U_{w}^{\pi} S_{w}^{\pi} V_{w}^{k T}$, $x=1,2, \ldots 256$ where $x$ represents the watermark block.

- Modify the singular values of each sub-band in the cover image with the singular values of each watermark block $S_{\hbar}^{x}=S_{\hbar}^{\pi}+a S_{i v}^{x}$ where $s_{\emptyset}^{x}$ denotes to the singular values of the cover sub-band $x, \mathbb{S}_{w}^{x}$ denotes to 
the singular values of the watermark block $x$, and $\alpha$ denotes to the scalar factor.

- Get 256 sets of modified wavelet packet coefficients, i.e., $\mathrm{I}^{\mathrm{I}}=\mathrm{U}_{\mathrm{b}}^{\mathbb{X}} \mathrm{S}_{\mathrm{b}}^{\mathrm{t} \mathrm{I}} \mathrm{V}_{\mathrm{b}}^{\mathrm{NT}}$ where $x=1,2, \ldots, 256$.

- Reconstruct the resultant sets of modified wavelet packet coefficients to get the watermarked image $I_{w}$.

2) Watermarking extraction: The watermark extraction is the reverse procedure of the watermark embedding. It can be summarized as follows:

- Use four-level Haar wavelet packet to decompose the watermarked image $I_{w}$ and the original image $I$ into 256 sub-bands in level 4.

- Apply SVD to each sub-band $I^{*}=U_{j}^{x} S_{j}^{x} V_{j}^{k T}$, $x=1,2, . ., 256$ where $x$ denotes to the sub-band.

- Extract the singular values from each sub-band $s_{w}^{x}=\left(S_{b}{ }^{x}-s_{j}^{T}\right) / \alpha$.

Construct the watermark blocks using the singular vectors $W^{x}=U_{w}^{k} s_{w}^{*} V_{w}^{* T}, x=1,2, \ldots, 256$ where $x$ points to the watermark block.

\section{PSO-based Watermarking Using WPT-SVD}

The role of the particle swarm optimization is to find the most appropriate wavelet packet sub-bands from the search space which includes all possible combinations of the WPT sub-bands and watermark blocks. Fig.3. shows the block diagram of PSO-based watermarking method using WPTSVD.

1) Watermarking embedding: The watermark embedding process goes through the following steps:

- Use the four-level Haar wavelet packet to decompose the cover image $I$ into 256 sub-bands in level 4.

- Apply SVD to each sub-band $I^{x}=U_{b}^{x} S_{b}^{x} V_{b}^{x T}$ $, x=1,2, \ldots 256$ where $x$ represents the cover image subband.

- Divide the watermark image into 16 non-overlapped blocks: $W=W^{l}+W^{2}+\ldots+W^{16}$, where $W^{x}$ denotes to the watermark block.

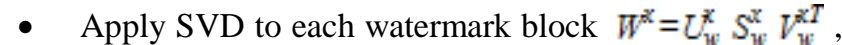
$x=1,2, \ldots 256$ where $x$ represents the watermark block.

- Modify the singular values of the selected sub-band by PSO in the cover image with the singular values of each watermark block $S_{\delta}^{x}=S_{j}^{x}+a S_{v}^{\pi}$ where $S_{j}^{x}$ denotes to the singular values of the cover sub-band $x$, $\mathbb{S}_{w}^{x}$ denotes to the singular values of the watermark block $x$, and $\alpha$ denotes to the scalar factor.

- Get 16 sets of modified wavelet packet coefficients, i.e., $I^{x}=U_{b}^{\pi} s_{b}^{\pi} V_{b}^{* T}$ where $x=1,2, \ldots, 16$ denotes to the indices of the selected sub-bands.

- Reconstruct the resultant sets of modified wavelet packet coefficients to get the watermarked image $I_{w}$.

2) Watermarking extraction: The watermarking extraction is the reverse procedure of the watermark embedding. It can be summarized as follows:

- Use four-level Haar wavelet packet to decompose the watermarked image $I_{w}$ and the original image $I$ into 256 sub-bands in level 4.

- Apply SVD to the sub-band selected by PSO $I^{*}=U_{b}^{*} S_{b}^{*} V_{b}^{* T}, \mathrm{x}=1,2, \ldots, 16$ where $\mathrm{x}$ denotes to the indices of the selected sub-band.

- Extract the singular values from the sub-bands that selected by the PSO in the embedding process by applying: $S_{w}^{x}=\left(S_{b}^{x}-S_{y}^{x}\right) / \alpha$ where $x$ represents the indices of the selected sub-band, $x=1,2, \ldots, 16, S_{\xi}^{x}$ denotes to the singular values of the watermarked sub-band and $S_{b}^{\text {th }}$ denotes to the coefficients of the cover image subband.

- Construct the watermark blocks using the singular vectors $W^{x}=U_{w}^{x} s_{w}^{x} V_{w}^{* I}, x=1,2, \ldots, 16$ where $x$ points to the watermark block.

\section{EXPERIMENTAL RESULTS}

The proposed watermarking techniques have been implemented in Matlab with Windows 7 enterprise edition environment.

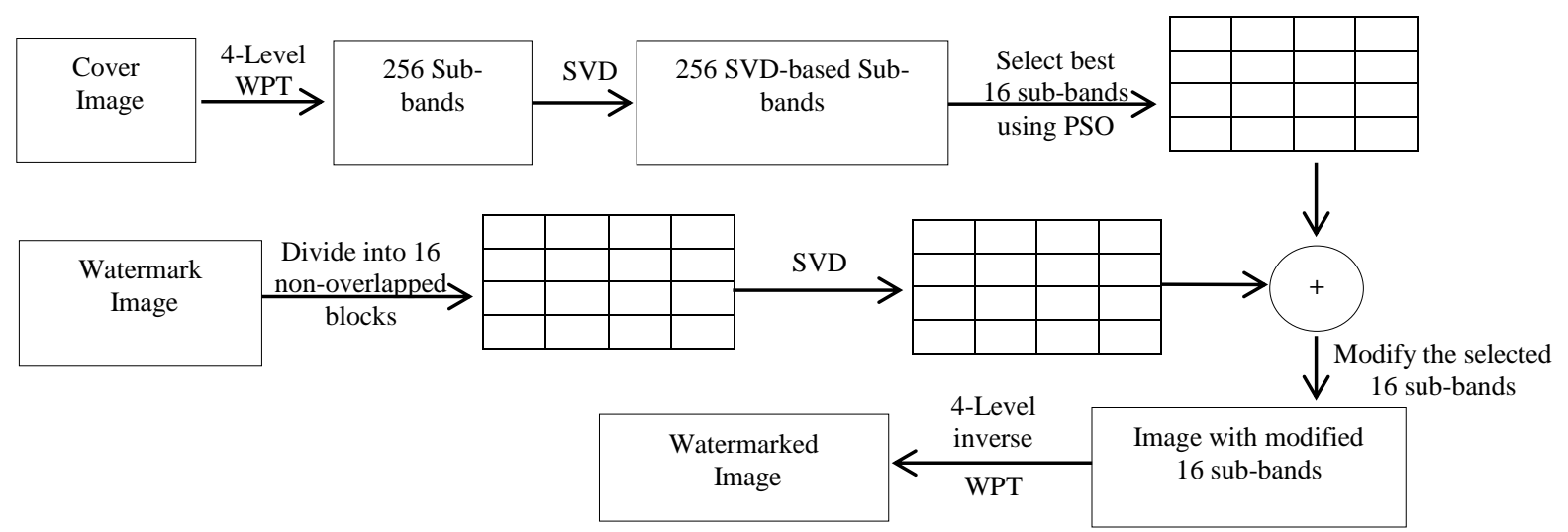

Fig.3. Block diagram of PSO-based Watermarking method using WPT-SVD. 
MATLAB supports built in numerous wavelets, MATLAB's Haar wavelet was adopted in the presented approaches. The performance of the watermarking algorithm can be measured in terms of imperceptibility and robustness to different types of intentional attacks and signal processing attacks. Many experiments are performed for the sake of evaluating the proposed approaches. The amount of hidden data is maximized in the cover images by making the embedded watermark image's size is equal to cover image size. The size of both cover image and watermark image is equal to $512 \times 512$. To evaluate the imperceptibility requirement of the proposed methods, the peak signal-to noise ratio (PSNR) metric is used. Although the Bit Correct Rate (BCR) and Normalized Correlation Coefficient (NCC) are used to measure the robustness requirement [22], the PSNR between the original watermark and the extracted watermark is used to measure the robustness. For image size of $N \times N$, the PSNR is calculated using (6).

As stated in the previous sections, the three techniques, WPT, WPT-SVD and PSO-based WPT-SVD are used. So the quality of the produced watermarked images using the three mentioned techniques are compared. Fig. 4. shows in the first row, the original cover image (Lena) along with the original watermark image. The second row shows the produced watermarked image using WPT, WPT-SVD and PSO-based WPT-SVD from the left to the right. The values of the peak signal to noise ratio measure are $65.99,65.99$ and 78.66 for WPT, WPT-SVD and PSO-based WPT-SVD respectively.

Fig.5. shows the imperceptibility between the original watermark image and the extracted watermark using the three proposed techniques. The first row of fig.5 shows the original cover image (Lena) along with the original watermark image, while the second row shows the extracted watermark from the left to right using WPT, WPT-SVD and PSO-based WPTSVD. The values of PSNR measure were 295.67, 298.13 and 320.08 for WPT, WPT-SVD and PSO-based WPT-SVD respectively.

Fig.6 shows the resistivity of the three proposed techniques to salt and paper (SP) attack applied on the produced watermarked image. The first row shows the original cover image (Lena) along with the original watermark image, while the second row presents the extracted watermark using WPT, WPT-SVD and PSO-based WPT-SVD from right to left. As depicted by Fig. 6, the WPT technique can be considered as fragile to the salt and noise attack since the extracted watermark has very high distortion. On the other hand, the extracted watermark image using WPT-SVD technique has small distortion which means that the employing of SVD technique gives the algorithm some resistivity to the attacks. The bottom right image in Fig. 6 which is the extracted watermark using the PSO-based WPT-SVD is expected to be better than the previous two due to the role of the PSO algorithm as an optimization algorithm. Regarding the salt and paper noise (SP) attack, the values of the peak signal to noise ratio (PSNR) measure are 6.49, 18.13 and 38.62 for WPT, WPT-SVD and PSO-based WPT-SVD respectively. Additional attacks referred to in [22] such as Median (MED) filtering, Histogram Equalization (HE), Motion Blurred (MB), Gaussian noise (GN) are applied to the watermarked images using

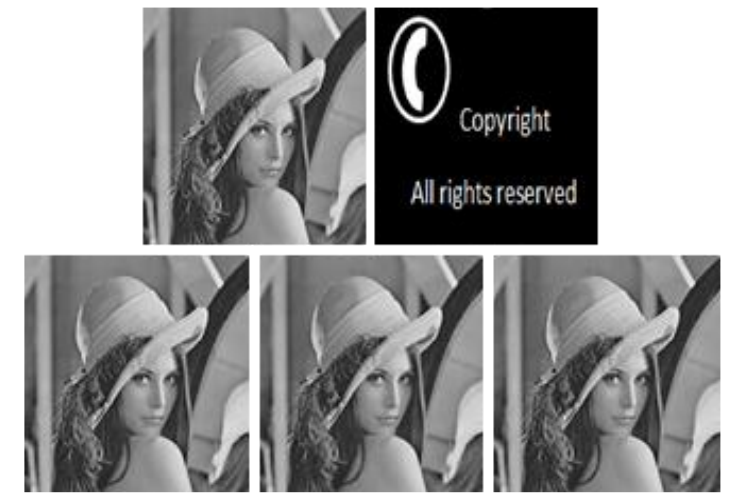

Fig.4. First row shows the original cover image and the watermark image. Second row shows the watermarked images using WPT, WPT-SVD and PSO-based WPT-SVD methods. The PSNR between the cover image and the watermarked image are $65.99,65.99$ and 78.66 respectively.

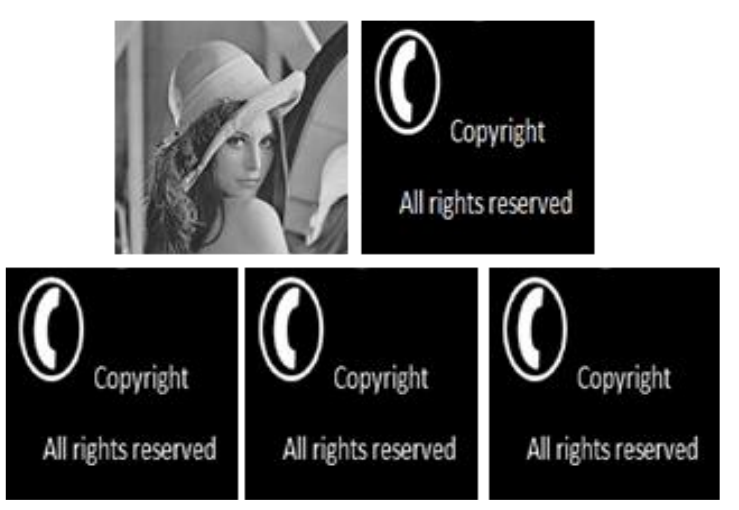

Fig.5. First row shows the original cover image and the watermark image. Second row shows the extracted watermark images using WPT, WPT-SVD and PSO-based WPT-SVD methods. The PSNR between the original watermark image and the extracted watermark image are 295.67, 298.13 and 320.08 respectively.

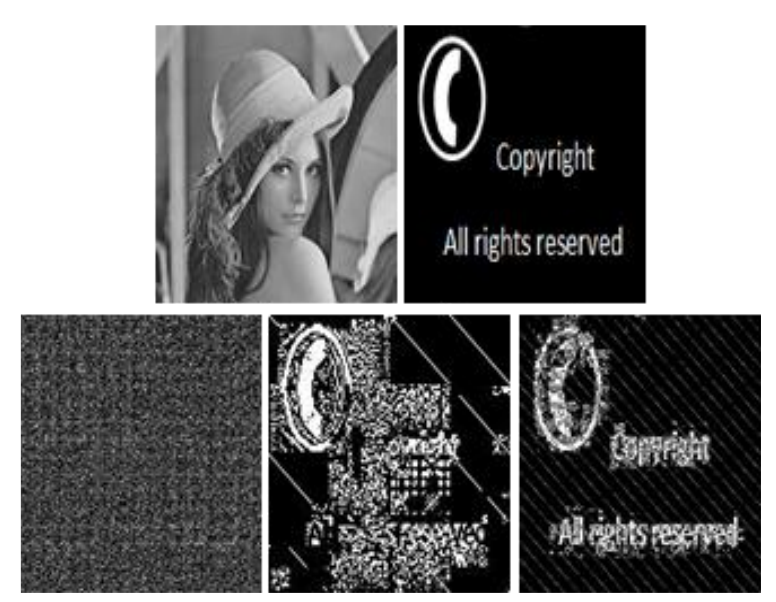

Fig.6. First row shows the original cover image and the watermark image. Second row shows the extracted watermark images of the attacked watermarked image using WPT, WPTSVD and PSO-based WPT-SVD methods. The PSNR between the original watermark image and the extracted watermark image are $6.49,18.13$ and 38.26 respectively. 
TABLE1. THE RESULTS OF IMPERCEPTIBILITY AND ROBUSTNESS OF THE PROPOSED WATERMARKING TECHNIQUES WITH DIFFERENT TYPES OF ATTACKS.

\begin{tabular}{|c|c|c|c|c|c|c|}
\hline \multirow{2}{*}{ Attack } & \multicolumn{2}{|c|}{ WPT } & \multicolumn{2}{c|}{ WPT-SVD } & \multicolumn{2}{c|}{ PSO-based } \\
\cline { 2 - 7 } & IMP & $\boldsymbol{R O B}$ & $\boldsymbol{I M P}$ & $\boldsymbol{R O B}$ & $\boldsymbol{I M P}$ & $\boldsymbol{R O B}$ \\
\hline SP & 65.99 & 6.49 & 65.99 & 18.13 & 73.21 & 38.62 \\
\hline GN & 65.99 & 7.26 & 65.99 & 15.47 & 78.68 & 49.25 \\
\hline MED & 65.99 & 5.25 & 65.99 & 13.93 & 78.64 & 50.31 \\
\hline HE & 65.99 & 3.58 & 65.99 & 8.70 & 78.65 & 31.43 \\
\hline MB & 65.99 & 4.38 & 65.99 & 12.29 & 78.67 & 31.57 \\
\hline
\end{tabular}

Matlab built-in functions. The results presented in table 1 show that applying the SVD technique enhanced the robustness of watermark algorithm due to the inherent properties of such technique. Additionally, applying PSO algorithm to the proposed technique participate in increasing the robustness effectively as presented in the table 1 for all types of attacks. The results of both imperceptibility (IMP) and robustness (ROB) are presented in table 1 .

In general, table 1 shows that, the proposed technique is more robust against salt and paper noise, Gaussian noise and median filtering attacks than histogram equalization and motion blurring attacks.

\section{CONCLUSION AND FUTURE WORK}

Imperceptibility, capacity and robustness are considered the most important requirements of watermarking techniques. Some of the watermarking applications require robustness and imperceptibility (quality) like military and medical imagery, while other applications such as those related to integritycontrol need high capacity. Therefore, a high capacity watermarking approach based on wavelet packet transform is presented. Also the concept of singular values decomposition is exploited to increase the resistivity to image processing attacks. Embedding watermarks into images can be considered as a constrained optimization problem. So, particle swarm optimization is used to solve this problem in this paper. PSO is used to select appropriate sub-bands from the permissible subbands for the sake of increasing both imperceptibility and robustness of the proposed watermarking algorithm. Experimental results prove that the proposed technique can increase the capability for resisting some image processing attacks along with the imperceptibility of the watermarked images if an appropriate PSO fitness function is adopted.

As future work, there still exist need for developing new optimal watermarking techniques to tradeoff among the watermarking requirements like high capacity, imperceptibility and robustness.

\section{REFERENCES}

[1] I. J. Cox, J. Kilian, F. T. Leighton, and T. Shamoon, "Secure spread spectrum watermarking for multimedia," Image Processing, IEEE Transactions on, vol. 6, pp. 1673-1687, 1997.
[2] T. V. Nguyen and J. C. Patra, "A simple ICA-based digital image watermarking scheme," Digital Signal Processing, vol. 18, pp. 762-776, 2008 .

[3] I. J. Cox, M. L. Miller, J. A. Bloom, and C. Honsinger, Digital watermarking vol. 53: Springer, 2002.

[4] R. Chamlawi and A. Khan, "Digital image authentication and recovery: Employing integer transform based information embedding and extraction," information Sciences, vol. 180, pp. 4909-4928, 2010.

[5] I. Cox, M. Miller, J. Bloom, J. Fridrich, and T. Kalker, Digital watermarking and steganography: Morgan Kaufmann, 2007.

[6] A. M. Ahmed and D. D. Day, "Applications of the naturalness preserving transform to image watermarking and data hiding," Digital Signal Processing, vol. 14, pp. 531-549, 2004.

[7] F. Hartung and M. Kutter, "Multimedia watermarking techniques," Proceedings of the IEEE, vol. 87, pp. 1079-1107, 1999

[8] M. Barni, F. Bartolini, A. De Rosa, and A. Piva, "Optimum decoding and detection of multiplicative watermarks," Signal Processing, IEEE Transactions on, vol. 51, pp. 1118-1123, 2003

[9] A. Briassouli and M. G. Strintzis, "Locally optimum nonlinearities for DCT watermark detection," Image Processing, IEEE Transactions on, vol. 13, pp. 1604-1617, 2004.

[10] A. Nikolaidis and I. Pitas, "Asymptotically optimal detection for additive watermarking in the DCT and DWT domains," Image Processing, IEEE Transactions on, vol. 12, pp. 563-571, 2003.

[11] P. Mangaiyarkarasi and S. Arulselvi, "A new digital image watermarking based on Finite Ridgelet Transform and extraction using ICA," in Emerging Trends in Electrical and Computer Technology (ICETECT), 2011 International Conference on, 2011, pp. 837-841.

[12] A. Ding and S. Dong, "Algorithm of Digital Image Watermark Based on Decomposition of Wavelet Packet," in Proceedings of the 2012 Sixth International Conference on Internet Computing for Science and Engineering, 2012, pp. 135-137.

[13] D. Coltuc, "Improved embedding for prediction-based reversible watermarking," Information Forensics and Security, IEEE Transactions on, vol. 6, pp. 873-882, 2011.

[14] J. Tian, "Wavelet-based reversible watermarking for authentication," in Electronic Imaging 2002, 2002, pp. 679-690.

[15] Y. Yan, Y. Sun, H. Q. Wang, Y. Lin, and H. Y. Chen, "Reversible authentication scheme of images based on lifting wavelet transform," in Applied Mechanics and Materials, 2013, pp. 1379-1382.

[16] G. Bhatnagar and B. Raman, "Wavelet packet transform-based robust video watermarking technique," Sadhana, vol. 37, pp. 371-388, 2012.

[17] Z. Wenyin and F. Y. Shih, "Semi-fragile spatial watermarking based on local binary pattern operators," Optics Communications, vol. 284, pp. 3904-3912, 2011

[18] H.-C. Huang and Y.-H. Chen, "Application of Genetic-Based Wavelet Packet Watermarking for Copyright Protection," in Recent Advances in Information Hiding and Applications, ed: Springer, 2013, pp. 139-153.

[19] A. Khan and S. A. Malik, "A high capacity reversible watermarking approach for authenticating images: Exploiting down-sampling, histogram processing, and block selection," information Sciences, vol. 256, pp. 162-183, 2014.

[20] A. H. Paquet, S. Zahir, and R. K. Ward, "Wavelet packets-based image retrieval," in Acoustics, Speech, and Signal Processing (ICASSP), 2002 IEEE International Conference on, 2002, pp. IV-3640-IV-3643.

[21] A. H. Paquet, R. K. Ward, and I. Pitas, "Wavelet packets-based digital watermarking for image verification and authentication," Signal processing, vol. 83, pp. 2117-2132, 2003.

[22] A. Furqan and M. Kumar, "Study and Analysis of Robust DWT-SVD Domain Based Digital Image Watermarking Technique Using MATLAB," in Computational Intelligence \& Communication Technology (CICT), 2015 IEEE International Conference on, 2015, pp. 638-644.

[23] Y.-R. WANG, W.-H. LiN, AND L. YANG, "AN INTELLIGENT WATERMARKING METHOD BASED ON PARTICLE SWARM OPTIMIZATION," EXPERT SYSTEMS WITH APPLICATIONS, VOL. 38, PP. 8024-8029, 2011. 